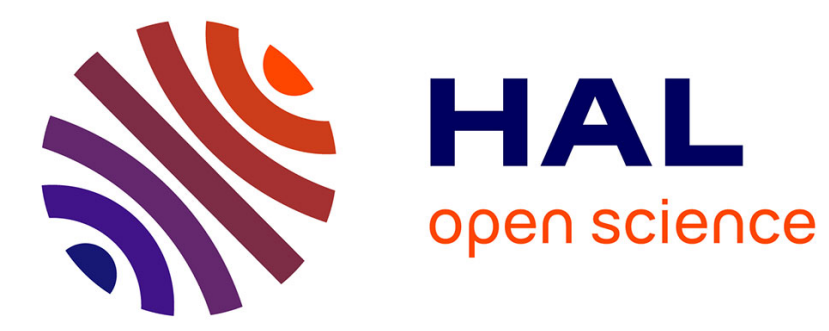

\title{
Explorer les mondes du risque dans la métropole São Paulo
}

\author{
Jacques Lolive, Cintia Okamura
}

\section{To cite this version:}

Jacques Lolive, Cintia Okamura. Explorer les mondes du risque dans la métropole São Paulo. Cahiers de géographie du Québec, 2018, 62 (177), pp.423-443. halshs-02826015

\section{HAL Id: halshs-02826015 https://shs.hal.science/halshs-02826015}

Submitted on 7 Jun 2020

HAL is a multi-disciplinary open access archive for the deposit and dissemination of scientific research documents, whether they are published or not. The documents may come from teaching and research institutions in France or abroad, or from public or private research centers.
L'archive ouverte pluridisciplinaire HAL, est destinée au dépôt et à la diffusion de documents scientifiques de niveau recherche, publiés ou non, émanant des établissements d'enseignement et de recherche français ou étrangers, des laboratoires publics ou privés. 
Cahiers de géographie du Québec

vol. $62, n^{\circ} 177$, décembre 2018

Pages 423-443

ARTICLE

\title{
Explorer les mondes du risque dans la métropole São Paulo
}

\author{
Jacques LOLIVE* \\ CNRS, PACTE, Grenoble \\ jacques.lolive@umrpacte.fr
}

\section{Résumé}

Comment vivre dans l'immense métropole du Brésil qu'est São Paulo, où les risques sont omniprésents? Comment restituer la diversité des situations de risque où se trouvent plongés les habitants? Comment analyser ce que vivre avec le risque veut dire, en restituant l'expérience des habitants et leur capacité d'action? Pour commencer à répondre à ces questions, nous utiliserons la théorie des mondes sociaux afin d'analyser trois situations de risque emblématiques de villes faisant partie de la métropole: Cajamar, une ville industrielle déstructurée composée de favelas; São Sebastião, une ville littorale sous l'emprise du plus grand terminal pétrolier d’Amérique du Sud; et le Condominío Barão de Mauá, un ensemble résidentiel construit sur une zone contaminée. Notre recherche révèle un processus contradictoire. D'une part, le risque façonne un milieu de vie qu'il dégrade souvent. D'autre part, les efforts multiformes des habitants se déploient pour maintenir et développer l'habitabilité d'un milieu de vie transformé par la production sociale des risques.

\section{Mots-clés}

Mondes sociaux, pragmatisme, société du risque, São Paulo, milieu de vie, culture du risque.

* Les deux auteurs ont une contribution équivalente dans l'élaboration du présent article.

\author{
Cintia OKAMURA* \\ CETESB, São Paulo \\ cintiaokamura@hotmail.fr

\section{Exploring risk management in the São Paulo metropolis}

\section{Abstract}

How can one live in the immense São Paulo metropolis where risks are omnipresent? How can one restore the diversity of risky situations experienced by the residents? How can one analyze what living with risk means by emphasizing inhabitants' experience and agency? To begin to answer these questions, we will use the social worlds theory to analyze three emblematic risk situations of cities within the metropolis: Cajamar, an unstructured industrial city composed of favelas; São Sebastião, a coastal town dominated by the first oil terminal in South America; and Condominío Barão de Mauá, a residential complex built on a contaminated area. Our research reveals a contradictory process. Risk shapes the living environment and degrades it more often than not. Meanwhile, residents make multifaceted efforts to maintain and develop the livability of that environment which has been transformed by the social production of risk.

\section{Keywords}

Social worlds, pragmatism, risk society, São Paulo, living environment, risk culture.
Explorar los mundos de riesgo en la metrópolis de San Pablo, Brasil

\section{Resumen}

¿Cómo vivir en la inmensa metrópolis brasileña, San Pablo, donde los riesgos son omnipresentes? ¿Cómo restablecer la diversidad de situaciones de riesgo en las que están enterrados sus habitantes? ¿Cómo analizar lo que vivir con riesgo significa, restituyendo la experiencia y la capacidad de acción de los habitantes? A fin de responder a esas preguntas, utilizaremos la teoría de los mundos sociales para analizar tres situaciones de riesgo simbólicas de las ciudades que hacen parte de la metrópolis: Cajamar, ciudad industrial desorganizada compuesta de favelas; San Sebastián, ciudad litoral sometida a la más grande terminal petrolera de América del Sur y, el condominio Barao de Mauá, conjunto residencial construido en zona contaminada. Nuestra investigación muestra un proceso contradictorio. Por un lado, el riesgo configura un medio de vida que a menudo se degrada. Por otro, los esfuerzos multiformes de los habitantes se despliegan para mantener y desarrollar la habitabilidad de un medio de vida transformado por la producción social de riesgos.

\section{Palabras claves}

Mundos sociales, pragmatismo, sociedad de riesgo, San Pablo, medio de vida, cultura del riesgo. 


\section{Introduction}

Avec ses 3181 zones contaminées (CETESB, 2017) remplies de zones d'habitation, d'écoles et de centres commerciaux; avec ses zones industrielles situées au milieu des quartiers d'habitations; ses 2,16 millions de personnes (IBGE, 2011) qui habitent des favelas aux dépens de toute considération de salubrité, d'hygiène ou de sécurité; avec ses inégalités sociales extrêmes; sa pollution atmosphérique; et ses rios transformés en égouts à ciel ouvert, la métropole du Brésil, São Paulo, constitue une véritable capitale du risque $^{1,2}$. La grande diversité des situations de risque qui imprègnent chacun des aspects de la vie de ses habitants fait de cette métropole un terrain privilégié d’analyse et d'expérimentation pour une recherche sur les risques. Pour réfléchir sur cette omniprésence du risque qui transforme les modes de vie des habitants de São Paulo, nous sommes partis de deux questions principales: Comment analyser la diversité des situations de risque dans la région métropolitaine de São Paulo (RMSP) tout en respectant ce caractère global qui marque chaque dimension de la vie humaine individuelle? Comment analyser ce que vivre avec le risque veut dire, en restituant l'expérience des habitants exposés, leur culture du risque et leur capacité d'action? L'analyse des mondes sociaux, élaborée par les sociologues de l'école de Chicago, sera utilisée pour aborder ces questions, une approche qui sera présentée dans l'énoncé de la problématique. Celle-ci s'articule autour de trois réflexions complémentaires correspondant aux deux questions précédentes.

Réflexion 1-Quelles relations entre risque et société satisfont à la fois des exigences de globalité et de diversité? Nous partirons de la notion de «société du risque» (Beck, 2001) qui permet une réflexion générale sur les relations entre le risque et la société dans nos sociétés contemporaines. Cependant, cette notion ne nous permet pas de faire des analyses concrètes. Nous devons donc

1 Depuis cette étude approfondie de 2011, le nombre a sans doute augmenté, mais c'est le dernier recensement spécifique dont nous disposons.

2 La RMSP, également connue sous le nom de Grand São Paulo, regroupe 39 municipalités de la conurbation paulistana avec une population estimée à plus de 21,5 millions d'habitants (IBGE, 2018). C'est un ensemble cohérent qui regroupe la ville de São Paulo avec la région industrielle du grand $\mathrm{ABC}$ (Santo Andre, São Bernardo do Campo, São Caetano do Sul, Diadema, Mauá, Ribeirão Pires et Rio Grande da Serra) et la majeure partie des zones périphériques d'habitations populaires. la compléter par la notion de «monde social» (école de Chicago reprise par Céfaï, 2015) qui permet de restituer la cohérence de chacune des situations de risque et nous fournit trois dimensions d'analyse (milieu de vie, risque collectif et culture) que nous utiliserons pour restituer la diversité des trois situations de risque étudiées (Cajamar, São Sebastião et Condominío Barão de Mauá).

Réflexion 2 - Quelles relations entre risque et culture favorisent l'action des habitants? Nous partirons de la notion de «culture du risque» (Douglas et Wildawski, 1983) qui définit une typologie des manières d'être, de faire et de penser le risque en fonction de la position sociale des individus. Cependant, cette notion ne tient pas compte des stratégies individuelles et collectives que les habitants sont susceptibles d'inventer pour améliorer leur situation. Nous utiliserons donc aussi les notions de «culture des problèmes publics» (Gusfield, 2007) et de «publicisation» (Dewey, 1927/2010) pour redéfinir la culture du risque comme un processus d'enquête collective visant la transformation d'une situation problématique, en l'occurrence les transformations du milieu de vie par le risque.

Réflexion 3-Quelles relations y a-t-il entre risque et expérience? Nous partirons de la notion de «milieu de vie» (Berque, 2000) comme transformation de l'environnement par l'humain pour en faire un monde de significations accessible par l'expérience vécue, sensible et affective. Ce processus de coconstruction du monde et de l'être humain (Hoyaux, 2002) explique le "concernement» (Brunet, 2008) de l'habitant, sa sensibilité orientée vers son milieu de vie, lorsque des transformations importantes, imposées et subies de ce milieu, génératrices de risque, suscitent des tensions et favorisent un comportement plus ou moins actif susceptible de prendre place dans un processus de mobilisation visant à résoudre ces tensions. Nous utiliserons la notion pragmatiste "d'enquête» (Dewey, 1993) pour redéfinir l'expérience vécue de concernement (lorsque le milieu de vie est transformé par le risque) comme une modalité de publicisation afin de pouvoir l'intégrer dans une démarche collective de recherche menée par les chercheurs et les habitants.

Notre problématique est également encadrée par deux réflexions épistémologiques.

Réflexion 4-Quel posture par rapport à la notion-concept de risque est compatible avec notre problématique? La notion de «société du risque» nous offre la matière d'une réflexion sur la notion de risque et de ses paradoxes qui nous interdisent 
tout positionnement exclusif formaté par le dualisme moderne, qu'il s'agisse du naturalisme, du constructivisme social ou du culturalisme. Le risque relève d'un positionnement intermédiaire, «trajectif» (Berque, 2000), qui n'est ni proprement objectif, ni proprement subjectif, mais procède d'une mise en relation des deux.

Réflexion 5-Quelle posture de recherche est compatible avec notre problématique? Nous partirons de la posture de «recherche-action» (Lewin, 1946) qui associe acteurs sociaux et chercheurs, volonté de changement et intention de recherche, coproduction des savoirs et contextualisation des connaissances. Cependant, dans un souci de cohérence avec les autres réflexions de la problématique, nous l'enrichirons avec la position pragmatiste d'engagement (Dewey, 1993) du chercheur en sciences sociales dans les processus de transformation de la société, en l'occurrence, dans les processus de publicisation des problèmes de risque.

Après avoir exposé le canevas argumentatif de notre problématique pour en montrer la cohérence et souligner l'apport de chaque référence théorique, nous allons maintenant présenter plus en détail chacune des réflexions problématiques.

\section{La théorie des mondes sociaux pour opérationnaliser la société du risque (réflexion 1)}

La théorie de la société du risque (Beck, 2001) permet de restituer l'omniprésence du risque dans la RMSP. Elle repose sur cinq thèses.

\section{La production sociale des risques environnementaux}

Selon Beck (2001), les risques contemporains ne viennent plus seulement de l'extérieur (catastrophes naturelles): ils sont engendrés par la société elle-même. Nous devons exercer notre réflexivité, c'est-à-dire examiner nos propres pratiques et les réviser, pour pouvoir gérer les risques inhérents à notre maîtrise de la nature, comme le montre la problématique cruciale du changement climatique, par exemple. Les nouveaux risques environnementaux sont les résultats non intentionnels des interventions de l'homme dans la nature.

\section{Des risques émergents, envahissants et controversés}

Ces risques nouveaux sont des «risques émergents» pour lesquels les effets sur la santé des humains et l'environnement sont mal connus. Ils sont envahissants par un effet de domino, qui désigne le risque multiplicateur constitué par la présence sur un même site de plusieurs établissements à risque ou par la possible combinaison de risques industriels et naturels lors d'un événement. Ce caractère proliférant des risques émergents signale leurs causes multiples et leurs conséquences imprévisibles. Les risques émergents sont donc caractérisés par un haut degré de complexité, d'incertitude et d'ambiguïté.

\section{La perception du risque fait partie de la définition du risque}

La société du risque brouille l'opposition tranchée entre la connaissance objective du risque, maîtrisée par les scientifiques, et la perception subjective du public, qui pèche à la fois par manque d'information sur la réalité du risque et par des représentations individuelles et collectives qui sous-estiment ou surestiment les éléments de cette réalité. La perception de la population exposée est partie prenante dans la définition du risque, car les menaces que cette population considère comme probables, susceptibles de devenir réalité, sont réelles par les conséquences qu'elles produisent. Ainsi, même un accident sans danger significatif pour la vie de la population locale (par exemple, l'incendie spectaculaire mais sans gravité d'une cuve de pétrole) pourrait entraîner un comportement de panique des habitants qui fuiraient en désordre. Ce comportement pourrait causer plus de blessés ou de morts que l'accident lui-même. Des chercheurs ont analysé comme étant une «amplification sociale du risque (Kasperson et al., 1988) le phénomène général correspondant à une telle situation. La perception du risque peut s'analyser comme un réseau de communication où le risque, qu'il soit calcul ou événement réalisé, est repris, analysé, transformé, métamorphosé par ceux qui s'en emparent parce qu'ils sont affectés, intéressés, ou en subissent les impacts.

\section{Le caractère mondial des nouveaux risques}

Les nouveaux risques ne peuvent plus être pensés comme des phénomènes locaux. Certains sont devenus de plus en plus dramatiques et globaux. Le réchauffement climatique et le risque nucléaire menacent toutes les régions du globe. De même, la situation de métropole «insoutenable» (au sens écologique) n'est pas l'apanage de São Paulo; on la retrouve dans la plupart des métropoles des pays du Sud et des pays émergents. Au niveau mondial, compte tenu du poids grandissant des pays du Sud dans l'urbanisation, il est vraisemblable de penser que la métropole du futur ne sera pas composée d'écoquartiers, comme ce qu'on anticipe pour les métropoles du Nord, mais qu'elle sera 
envahie par le «bidonville global», telle une amplification des problèmes actuels de São Paulo.

\section{L'inégale répartition sociale des risques}

Bien que le risque soit devenu un phénomène global, la répartition sociale des niveaux d'exposition reste inégale. Ainsi, les habitants des favelas sont plus exposés que les habitants des condominiums de luxe à une combinaison inédite de risques variés (inondations, éboulements, accidents industriels, pollution multiforme, etc.).

Cette théorie de la société du risque a fait l'objet de nombreuses critiques. Pour le sociologue Alain Bourdin (2003) qui les présente dans son article, c'est un concept trop large, un discours général convaincant, mais qui peine à restituer la pluralité des situations. Nous partageons son opinion. Pour opérationnaliser ces analyses de la société du risque tout en restituant la diversité des situations de risque dans la métropole São Paulo et leur caractère englobant, nous utiliserons le concept de «monde social» remis à l'ordre du jour par le sociologue Daniel Céfaï (2015), qui définit le monde social à partir de trois notions: le milieu de vie, le champ d'activités collectives et le modèle expérimental de la culture.

\section{Le milieu de vie}

Le monde social est une niche écologique. La société des humains n'est pas du même type que celle des animaux parce qu'elle transforme beaucoup plus son environnement naturel en environnement social et culturel, et qu'elle engendre son propre milieu de vie. Le sociologue George Herbert Mead (2006) nous invite à considérer la dimension écologique de cette transaction entre cet organisme social et ses environnements. Selon lui, ce monde social n'est pas complètement clos sur lui-même, mais il s'ouvre par des formes de solidarité écologique sur d'autres écosystèmes. Ce milieu de vie constitue un «habitat expérientiel» (Idem: 90), un champ d'expérience pour les humains. Pour transposer cela dans le champ du risque, nous analyserons comment le milieu de vie des habitants de São Paulo se transforme sous l'effet du développement des risques dans la métropole.

\section{Le champ d'activités collectives}

Les mondes sociaux sont constitués avant tout d'activités collectives. Les participants d'un monde social doivent équiper ce monde en objets, en outils et en instruments à concevoir, à fabriquer et à entretenir. Pour que cette organisation dure, ils stabilisent des activités conjointes, régulent des processus de coordination, les encadrent par des conventions de coopération et de compétition. Donc, pour comprendre un monde social, il faut comprendre comment ses multiples activités viennent s'imbriquer les unes dans les autres et se transforment au cours du temps. Le monde social amène donc l'idée d'une «activité conjointe, orientée vers des foyers d'attention commune et engageant un processus d'auto-organisation qui, progressivement, fait émerger objectifs et moyens » (Céfaï, 2015: 2). Le sociologue Howard Becker dit à peu près la même chose pour définir le monde de l'art: «Le monde de l'art correspond aux personnes et aux organisations qui produisent les événements et les objets que ce monde définit comme de l'art» (1976: 703). Ainsi, l'organisation du monde social s'effectue autour d'un centre d'intérêt en regroupant tous ceux qui sont concernés. Si l'on transpose cela au domaine du risque, le monde du risque regroupera les experts qui définissent le risque, les entreprises qui le produisent, les administrations qui le gèrent et les populations qui sont affectées par lui.

\section{Le modèle expérimental de la culture}

Chaque monde social est stabilisé par une culture. Ici, la culture n'est pas un système de représentations dans la tête des habitants, mais un ensemble de prémisses d'action impliquées dans des situations où se passent différents processus. Des hypothèses formulées par les habitants vont être confirmées ou infirmées par leurs expériences. Pour pouvoir transposer ce modèle expérimental de la culture au champ du risque, nous envisagerons la culture du risque comme un processus de publicisation. Nous allons développer cette idée dans notre seconde réflexion problématique.

\section{La publicisation pour que l'expérience habitante transforme la culture du risque (réflexion 2)}

Selon l'anthropologue Mary Douglas et le politologue Aaron Wildavsky, la culture du risque des acteurs dépend essentiellement de leur position sociale (Douglas et Wildavsky, 1983; Douglas, 1999). Douglas et Wildavsky définissent quatre types de culture:

- Ia culture individualiste et compétitive des industriels, du marché et des entrepreneurs, qui encourage les individus à prendre des risques, à investir, et s'inquiète de toute entrave à la liberté d'initiative de ses membres; 
- Ia culture hiérarchique et bureaucratique des administrations d'État, qui pratique la gestion calculée des risques afin de défendre l'intérêt du plus grand nombre et critique les représentations irrationnelles des associations et des habitants;

- la culture égalitariste et communautariste des mouvements de défense de l'environnement, que les auteurs qualifient de sectaires par comparaison au mode de vie des Amish; ces mouvements cherchent à gagner des adhérents en sonnant l'alarme au moindre danger et dénoncent les scandales qui corrompent la société et prouvent leur propre pureté, au risque de perdre le contact avec le monde réel;

- La culture fataliste des victimes et des dominés, qui se retrouvent isolés quand ils sont confrontés à des organisations et des structures complexes qu'ils ne comprennent pas. Ils pratiquent le déni du risque, le culte de la sécurité et la soumission aux menaces. Ils sont stigmatisés par les trois autres cultures.

Cette typologie des manières d'être, de faire et de penser le risque est corroborée par de nombreuses observations empiriques. Pour autant, ses interprétations plutôt conformistes, qui expliquent sans doute son grand succès chez les administrations et les entreprises, c'est-à-dire chez les représentants des deux cultures qu'elle valorise, sont remises en cause par la critique de l'anthropologue Tobias Girard (2013). Ce dernier s'appuie sur les objections de plusieurs auteurs pour dénoncer le biais néoconservateur de Douglas et Wildavsky (1983), leur penchant pour l'ordre social, l'autorité hiérarchique et le respect des règlements. Dans ces conditions, on comprend que cette grille ne s'intéresse pas vraiment à l'expérience du risque des habitants et aux stratégies individuelles ou collectives qu'ils sont susceptibles d'inventer pour améliorer leur situation. C'est la raison pour laquelle nous ne l'utiliserons pas telle quelle.

Pour apprécier l'intérêt de cette théorie, il faut la relire à la lumière des analyses du politologue Joseph Gusfield (2007) sur la culture des problèmes publics. «Pour Gusfield, la culture publique est une perspective commune, prise pour allant de soi, qui commande à la perception de ce qu'est l'ordre public et qui est transformée par l'émergence de nouveaux problèmes publics» (Céfaï, 2013: 12). Le politologue envisage les problèmes publics comme des objets théâtraux. Comme pour tout acte de représentation théâtrale, la mise en forme, la mise en scène des problèmes publics, a une signification symbolique. La construction des problèmes publics est toujours liée à un ordre moral, à une définition du bon et du mauvais dans un domaine de la vie sociale. On est frappé par la ressemblance de ces analyses avec celles de Douglas et Wildavsky qui caractérisent les cultures du risque selon des critères quasi moraux. Ces auteurs nous fournissent donc les principaux éléments de la culture des problèmes publics concernant les risques, mais ces éléments sont formulés d'une manière trop générale, sans que ces cultures puissent évoluer et sans réflexivité des auteurs concernant leur biais politique. C'est pourquoi, à notre avis, on confond ici les résultats provisoires d'un processus de publicisation avec une culture stable et établie.

À l'inverse, Joseph Gusfield ne s'intéresse pas seulement à la construction des problèmes publics par les policy makers, les individus et groupes sociaux légitimes. Sa grille est compatible avec les analyses de John Dewey centrées sur la formation d'un public. Si l'on fait converger les deux, on obtient l'analyse de la publicisation qui décrit la constitution d'un collectif autour d'un problème. Des personnes confrontées à une situation problématique éprouvent un trouble indéterminé. Le public émerge à travers les interactions de ces personnes qui vont se constituer en un collectif d'enquêteurs et monter des dispositifs de mobilisation pour définir leur trouble, le transformer en problème d'intérêt public et interpeller les pouvoirs publics pour qu'ils le résolvent. Nous analyserons la culture du risque comme un processus de publicisation amorcé par les populations exposées au risque, qui transforme les actions de l'État et modifie les cultures établies si bien définies par Douglas et Wildavisky. Cette publicisation est le support de la culture du risque, car elle permet de s'approprier le nouvel environnement des humains qu'est la société du risque afin d'y puiser des ressources pour des expériences individuelles et collectives impliquant la participation des individus et favorables à leur développement individuel. Ainsi, la culture du risque permet la réflexivité du monde du risque. Ce processus émergent s'enracine dans l'expérience habitante. Cette notion sera analysée dans notre troisième réflexion problématique. 


\section{Une posture de recherche pragmatiste pour rapprocher l'expérience des habitants de l'enquête des sciences sociales (réflexion 3)}

La géographie phénoménologique (Dardel 1990 ; Berque 2000; Hoyaux 2002) analyse l'habiter comme l'humanisation de l'environnement biophysique et objectif par la sphère du symbolique. "Habiter pour l'être-là, c'est donc se construire à l'intérieur d'un monde par la construction même de celui-ci» (Hoyaux 2002: 5). L'être humain construit son monde en agençant différents éléments qu'il a prélevés dans l'environnement par des choix perceptifs et des choix d'imagination. Ce processus révèle l'être humain à lui-même grâce aux significations qu'il produit par son faire, son dire, son penser. Ce monde de significations est accessible par l'expérience vécue, sensible et affective. Ces analyses nous permettent d'expliquer la formation du milieu de vie. Habiter, pour I'humain, c'est transformer l'environnement d'une manière qui lui soit propre pour en faire son milieu de vie (Berque, 2000). Le milieu de vie est constitué par la manière que nous avons d'appréhender les choses par nos sens, nos mots, nos pensées, nos actions. C'est la relation sensible, corporelle, pratique et imaginative qu'entretient l'habitant avec son environnement.

Ce processus de coconstruction du monde et de l'être humain (Hoyaux, 2002) explique pourquoi porter atteinte au milieu de vie, c'est du même mouvement affecter l'être qui s'est construit en s'y mouvant. L'humain est un être sensible, sensoriel et réflexif qui ne saurait être insensible à la destruction de ce milieu dans lequel il a appris à vivre. Le concernement (Brunet, 2008) s'enracine dans cette émotion de l'être humain qui répond à l'atteinte à son milieu de vie. Nous le définirons comme toute sensibilité de I'habitant orientée vers son milieu de vie, lorsque des transformations importantes, imposées et subies de ce milieu, génératrices de risque, suscitent des tensions et favorisent un comportement plus ou moins actif susceptible de prendre place dans un processus de mobilisation visant à résoudre ces tensions. Ainsi, le concernement produit un potentiel d'engagement, une disponibilité pour l'action collective.
La philosophie pragmatiste (Dewey, 1993) permet de redéfinir l'expérience vécue du milieu de vie et le concernement qui la prolonge pour pouvoir mieux les intégrer dans une démarche collective de recherche et de transformation sociale. Pour Dewey, cité par la philosophe Joëlle Zask (2008: 47) «une expérience consiste à joindre une séquence d'actions à quelque chose que l'on ressent, qui nous affecte». Cette phase d'examen correspond à une enquête. «Au cours de celle-ci, je tâche d'identifier les causes du trouble éprouvé, de les utiliser pour imaginer une hypothèse de résolution, de mettre cette hypothèse à l'épreuve, et ainsi de suite» (Ibid.). Ainsi le ressenti, la séquence affective, doit constituer le point de départ d'une enquête pour appartenir à l'expérience. L'enquête ainsi définie est ce que Dewey appelle «l'enquête de sens commun » (1993); c'est la relation d'adaptation, d'ajustement de nos comportements à l'environnement. Le public est analysé en tant que communauté d'enquêteurs. Dewey (1993) rapproche cette forme d'enquête de l'enquête scientifique, qui obéit à la même structure. Seules les motivations diffèrent. L'enquête de sens commun menée par les personnes concernées vise à satisfaire un besoin vital du quotidien, tandis que l'enquête scientifique menée par les chercheurs vise un problème de recherche plus global. Cependant, il ne faut pas exagérer cette différence. Un autre philosophe pragmatiste, William James (2005), «imagine une conception «additive» de la recherche, qui ne se substitue pas aux enquêtes et aux expérimentations menées par les enquêtés, ni prétende parler en leur nom, mais fasse émerger de nouvelles perspectives d'expérience et d'action en coopérant avec eux» (Céfaï et al., 2015: 10). L'enquête du chercheur doit s'inscrire dans la continuité d'une enquête décrite et menée par les personnes concernées, avec l'idée que «l'expérience des uns s'ajoute à l'expérience en cours des autres et la prolonge dans d'autres possibles» (Hennion, 2015: 9). Ainsi, enquête, expérience et expérimentation sont quasiment synonymes.

\section{Un positionnement trajectif sur la notion de risque (réflexion 4)}

Quel positionnement pouvons-nous adopter sur la notion de risque qui soit compatible avec notre problématique? Plutôt que de comparer théoriquement les différents positionnements sur le risque, nous avons préféré réfléchir à partir d'une notion, la société du risque. Comme Ulrich Beck (2001), nous utiliserons le terme de risque dans son usage courant, tel qu'il a été imposé par l'usage et le débat 
social: le risque c'est le danger possible, la menace de péril ou de catastrophe. Ce sens du mot risque est distinct de celui adopté par les spécialistes, qui le définissent en général comme «la combinaison de la probabilité d'occurrence d'un ou plusieurs événements dangereux ou de l'exposition à un ou à de tels événements et de la gravité du préjudice personnel ou de l'atteinte à la santé que cet événement ou cette/ces exposition(s) peuvent causer» (BS OHSHS, 2007). II offre cependant l'avantage de mieux correspondre à la perception du risque par les habitants. Ainsi, le géographe Emmanuel Martinais (2015) a étudié les contestations riveraines qui, depuis le tournant des années 2010, critiquent la politique de prévention des risques industriels. Pour les associations qui critiquent l'élaboration et la mise en œuvre des plans de prévention des risques technologiques, "l'approche probabiliste au fondement de la prévention permet d'écarter des phénomènes dangereux potentiellement très graves au motif que des dispositifs techniques de sécurité ont été mis en place pour réduire leur probabilité d'occurrence. Elle laisse finalement inchangés les dangers les plus importants qu'il faudrait réduire à la source» (Martinais, 2015: 27) en diminuant, par exemple, la quantité d'une substance dangereuse stockée dans les réservoirs, en déplaçant les bassins de stockage installés trop près des habitations voisines ou en les confinant dans des enceintes de béton. La notion de société du risque que nous utilisons dans cet article a le mérite de révéler les paradoxes constitutifs de la notion de risque qui ne permettent pas d'adopter une des positions épistémologiques exclusives formatées par le dualisme moderne (qui oppose sujet et objet) qu'il s'agisse du naturalisme, du constructivisme social ou du culturalisme. Pour les définir, nous nous référons à la présentation très claire des trois postures dans un article favorable à la seconde (Martinais et al., 2006).

À première vue, la société du risque ressort du constructivisme social puisqu'elle met l'accent sur la production sociale du risque par les sciences et les techniques. Le risque est envisagé comme «un construit, historiquement et socialement situé et doit être questionné comme tel» (Idem: 173). Cependant, la société du risque procède aussi d'une autre posture, «culturaliste», qui «s'intéresse davantage aux perceptions, attitudes et comportements des populations [...] et chercher en général à montrer que l'appréhension "ordinaire» du risque diffère de sa définition scientifique ou technique et à interpréter ce décalage en termes psychologiques, sociaux ou culturels » (Ibid.). Cependant, c'est un culturalisme sophistiqué puisque l'accent mis sur le rôle de la perception dans la définition du risque fait que la notion ressort plutôt du «constitutivisme» défini par le géographe AndréFrédéric Hoyaux (2015) comme autoconstitution du risque à travers l'invention de la définition même de ce que l'on considère comme tel. Ces perceptions performatrices vont susciter des actions variées qui vont formater le monde du risque.

Cette réflexivité constitutive du risque s'épanouit dans les analyses de la «modernité réflexive» réalisées par Beck et ses collègues Giddens et Lash (1994) pour qui le triomphe du système industriel brouille les limites entre nature et société, jusqu'à l'internalisation de la nature au processus industriel et à la civilisation. Nous sommes entrés dans une seconde modernité, réflexive, c'est-à-dire que l'essor des sciences et des techniques se poursuit, mais que ce processus ne peut plus être naïf. II nous demande de nous interroger, tant individuellement que collectivement, sur ce que nous sommes en train de faire, d'expérimenter.

Une des caractéristiques essentielles de cette modernité réflexive est le fait que nous devons gérer les risques inhérents à notre maîtrise-comme le montre la problématique cruciale du réchauffement climatique, par exemple. Nous sommes devenus maîtres et possesseurs de la Nature, comme le prévoyait Descartes, mais le produit de cette maîtrise, la Nature seconde profondément hybridée, transformée par l'action modernisatrice, nous échappe, comme en a témoigné la crue spectaculaire du Var, le 5 novembre 1994, à Nice. Cette crue a provoqué l'inondation de l'aéroport, de la cité administrative, du marché d'intérêt national, du quartier de Nice-Ouest, la rupture de la voie sur berge d'accès à l'aéroport, etc. Par chance, l'accident a culminé en soirée; il n'y a pas eu de victimes. Le débit de la crue a atteint les $3800 \mathrm{~m}^{3} / \mathrm{s}$ en aval du fleuve. II a largement dépassé la limite de $3000 \mathrm{~m}^{3} / \mathrm{s}$ que les experts attribuaient à la crue millénaire dont la probabilité d'apparition est très faible. Deux facteurs ont permis d'expliquer ce phénomène: I'urbanisation de la basse vallée du Var, bien sûr, mais aussi des aménagements successifs (endiguements, extractions, construction des seuils et des microcentrales, etc.) qui ont artificialisé le lit du Var. Pour s'adapter à ces perturbations anthropiques, le fleuve a remodelé sa morphologie: baisse de la nappe, «chenalisation» du lit, développement de la végétation, débordements en crue, enfoncement à l'aval, érosion, engravement des seuils amont, remontée du lit... Cela a exposé la vallée à des risques accrus d’inondation. 
Donc les risques de la modernité réflexive sont la forme méconnaissable de résultats non intentionnels des interventions de l'humain dans la nature. Cette méconnaissance du rôle de «l'humanisation» du fleuve dans le renforcement des risques d'inondation va paradoxalement alimenter un naturalisme basé sur la difficulté de maîtriser cet hybride. La gravité des crues et leur caractère imprévu suggèrent l'idée d'une autonomie d'action et d'une résistance caractéristique d'un phénomène naturel, et ils vont donc favoriser la «naturalisation» du risque, qui deviendra l'effet dudit phénomène naturel doté d’une objectivité indépassable.

Ainsi, la société du risque nous permet de réfléchir sur les limites des positionnements traditionnels concernant le risque. Le naturalisme n'est qu'un effet de l'hybridation, le constructivisme social néglige la capacité d'action de la nature humanisée, la perception du risque est constitutiviste et «performatrice». Le risque, tant dans son versant subjectif que dans son versant objectif ou social, procède en fait d'une réflexivité constitutive qui associe les domaines artificiellement séparés constitutifs de notre modernité, sujet et objet, nature et société (Latour, 1991). II relève d'un positionnement intermédiaire, «trajectif» (Berque, 2000), qui n'est ni proprement objectif, ni proprement subjectif, ni proprement social, mais procède d'une mise en relation des trois, laquelle contribue à son efficacité singulière. $\mathrm{Ce}$ caractère hybride du risque autorise l'utilisation d'un schéma actanciel: il nous permet de l'analyser comme un actant, un opérateur à la fois social, politique et naturel qui transforme et organise les mondes du risque.

\section{Une posture pragmatiste d'engagement du chercheur (réflexion 5)}

Quelle posture de recherche allons-nous adopter qui soit compatible avec notre problématique? Notre démarche de recherche s'inspire d'abord de la recherche-action, dont les principes ont été formulés à la fin des années 1940 par Kurt Lewin, un psychologue étasunien spécialisé dans la psychologie sociale. Pour lui, l'expérimentation est indispensable pour fonder une proposition scientifique même en sciences sociales. Préconisant qu'on ne peut comprendre la réalité à laquelle se réfère un concept qu'en agissant sur elle, Lewin est conduit à envisager une " expérimentation dans la vie courante », ce qui l'amènera à définir la recherche-action dont les principes sont énoncés par le sociologue consultant Guy Le Boterf:
La recherche-action est un processus dans lequel les acteurs sociaux ne sont plus considérés comme de simples objets passifs d'investigation, deviennent de plus en plus des sujets conduisant une recherche avec la collaboration de chercheurs professionnels. Ce sont donc les groupes sociaux concernés qui vont identifier les problèmes qu'ils veulent étudier, en réaliser une analyse critique et rechercher les solutions [...] Entre le chercheur ou l'expert et les acteurs s'établissent des relations de coopération et d'éducation mutuelle. Le chercheur apprend de l'expérience des acteurs, et les acteurs apprennent du chercheur des méthodologies et des techniques. Par le partage des activités de recherche qu'elle implique, la recherche-action remet en cause la traditionnelle division du travail entre les intellectuels et ceux qui, socialement, ne sont pas considérés comme tels (Le Boterf, 1983: 44).

Cette posture qui associe acteurs sociaux et chercheurs, volonté de changement et intention de recherche, coproduction des savoirs et contextualisation des connaissances nous convient. Cependant, dans un souci de cohérence avec les autres réflexions de la problématique, nous l'enrichirons avec la posture pragmatiste d'engagement (Dewey, 1993) du chercheur en sciences sociales dans les processus de transformation de la société. L'engagement des sciences sociales dans les processus de transformation de la société est une conséquence de cette posture pragmatique puisque l'enquête en tant que processus expérimental de base implique qu'il existe un lien fondamental entre l'enquête de sens commun et l'enquête scientifique. Une seconde raison qui motive cet engagement est que «pour être véritablement «claire» (c'est-à-dire prétendre au statut d'énoncé scientifique), il faut qu'une idée ait pris en compte les conséquences pratiques qu'elle est susceptible de produire, ce que seule l'expérimentation peut révéler » (Daynac, 2002: 3). Au lieu d'analyser la correspondance, l'adéquation entre une théorie et un objet d'étude figé dans le présent, le chercheur contribue aux transformations réciproques de la théorie et du terrain. Ceci requiert l'implication du chercheur dans l'évolution en cours.

Dans notre recherche, la prise en compte de cette exigence implique que nous analysions et favorisions la constitution d'une culture du risque redéfinie à partir d'un processus de publicisation des habitants quand ils sont confrontés au problème du risque. Nous accompagnerons et stimulerons la prise de conscience et la mobilisation des habitants en suivant une démarche en trois temps: d'abord en les sensibilisant aux dangers potentiellement 
présents sur les sites industriels; ensuite en examinant avec eux leur «vulnérabilité », c'est-à-dire la manière dont ces dangers et ces risques peuvent affecter les différents membres de leur communauté; enfin en leur proposant de faire vivre un forum du risque, un dispositif participatif composé de deux forums complémentaires appelés à travailler ensemble, l'un réunissant les habitants exposés et l'autre les différentes institutions concernées. Ce forum composite est mis en place pour débattre des informations disponibles et associer les habitants à la gestion du risque. En accord avec cette posture et cette démarche, nous privilégions l'expérimentation de méthodologies participatives ${ }^{3}$. Dans l'exposé, nous présenterons quelques-unes de ces méthodes et nous verrons comment elles doivent s'adapter aux spécificités des différents terrains de recherche:

- une méthodologie utilisée à Cajamar - la connaissance ambulatoire-s'adapte à un terrain difficile pour collecter des informations et entrer en contact avec un public en cours de constitution;

- deux méthodologies utilisées au Condominío Barão de Mauá - l'immersion anthropologique et les forums participatifs - s'adressent à un public déjà constitué pour partager son expérience du risque, chargée d'émotions, et l'impliquer dans la gestion du risque;

- une méthodologie utilisée à São Sebastião-le théâtre-forum - pour favoriser l'émergence d'un public autour d'une catastrophe technologique dont il fera l'expérience imaginaire.

Pour conclure sur notre transposition de l'analyse des mondes sociaux aux situations de risque, notons que cette problématique exigeante définit un cadre théorique, un référentiel et un programme d'action que nous n'avons pu réaliser qu'en partie compte tenu de l'incomplétude de nos recherches et de l'inachèvement des mondes du risque analysés. En effet, si le risque est un analyseur pour étudier la construction des mondes sociaux par les individus et groupes sociaux, nous n'analysons pas un monde social stabilisé, mais la possible constitution d'un monde social autour du risque. C'est un processus contradictoire. D'une part, le risque façonne un milieu de vie qu'il dégrade souvent. D’autre

3 Nous utilisons également des méthodes plus classiques pour recueillir des informations, qu'il s'agisse de données secondaires (les recensements de l'Instituto Brasileiro de Geografia e Estatística [IBGE], l'équivalent de l'Institut national de la statistique et des études économiques [INSEE] français) ou de données primaires (travail de terrain, entretiens semi-directifs avec les habitants des zones à risque). Mais cet article ne leur est pas consacré. part, les efforts multiformes des habitants maintiennent et développent l'habitabilité d'un milieu de vie transformé par la production sociale des risques. Pour étudier la formation d'un monde social du risque, nous analyserons donc comment la constitution d'un milieu de vie façonné par le risque, un quartier d'habitation exposé au risque par exemple, peut susciter la transformation des activités collectives et favoriser l'émergence d'une culture du risque.

Comme une bonne partie de la population métropolitaine habite dans un quartier de favelas ou dans une zone contaminée ou dans le voisinage immédiat d'une industrie dangereuse, nous allons analyser un exemple de chacune de ces situations de risque emblématiques de la métropole.

\section{Cajamar: repenser l'espace public pour un quartier de Favelas}

\section{Choisir une méthodologie adaptée pour un terrain difficile}

Cajamar est une ville de la métropole située à $30 \mathrm{~km}$ au nord de São Paulo. Notre recherche exploratoire visait à définir une stratégie de sensibilisation de la population exposée au risque de contamination des eaux de consommation. Confrontés à une ville qui nous apparaissait impénétrable dans sa confusion, sa violence et son apparence chaotique, il nous a fallu laisser de côté nos idées préconçues pour nous immerger dans cette ville et nous ouvrir à ses «sollicitations». Nous avons adopté une méthode d'analyse préalable, le «paradigme inquiet», définie par l'anthropologue italien Massimo Canevacci (1997) et retenu par Cintia Okamura. Sa méthode est basée sur le déracinement et la perte des repères familiers. Pour Canevacci (1997: 5), il faut «se perdre dans la ville», car le déracinement et «le rendre étrange $(r)$ » sont des moments essentiels pour atteindre de nouvelles possibilités cognitives. À travers les mélanges imprévisibles et fortuits entre les niveaux rationnels, perceptuels et émotionnels, il est possible d'atteindre le raffinement du regard urbain. Cette méthode est nécessaire, selon lui, pour interpréter la polyphonie des différents points de vue des habitants et des chercheurs qui lisent et interprètent la métropole dans laquelle ils coexistent. Une autre source d'inspiration importante a été la «connaissance ambulatoire» proposée par le philosophe pragmatiste William James (2005). Lapoujade (1997) résume les trois propositions qui définissent cette posture de recherche: 1) la connaissance est une déambulation: l'expérience du 
mouvement (le cheminement de proche en proche, de rencontre en rencontre, d'expérience en expérience) et le mouvement de la recherche sont liés; 2) le pluralisme: la ville éclatée est un archipel, une courtepointe où chacune des rencontres exprime un des petits mondes singuliers qui la composent; 3) le connexionnisme: la connaissance est une exploration et une mise en réseau de cette diversité.

Notre déambulation dans la ville s'est effectuée au hasard des rencontres, en cheminant d'expérience en expérience pour permettre qu'une ville informe, opaque et impénétrable n'offrant aucune prise à nos réflexions gagne en intelligibilité afin de devenir notre terrain d'analyse et d'intervention ${ }^{4}$. Certaines des personnes (par exemple, un élu passionné d'histoire, le propriétaire d'un bazar animateur d'un réseau social, une habitante de longue date d'une favela) que nous avons rencontrées alors ne se contentaient pas de jouer le rôle d'informateurs, elles devenaient des points d'accroche sur une dimension de la réalité cajamarense qui allait bousculer les réflexions et les croyances précédentes des chercheurs. Ces temps forts ont révélé l'existence de nouvelles relations qui ont permis un changement de regard, une clarification et une bifurcation de notre recherche. Parallèlement, ce parcours de recherche a transformé les chercheurs. Ils ont fini par s'attacher à cette petite ville ingrate et «mal aimée » qu'ils avaient tendance à rejeter au début de la recherche.

\section{Le développement incontrôlé d'une ville par les occupations irrégulières}

\section{Une ville éclatée}

Cajamar est une ville de 100000 habitants éclatée entre trois sites: Cajamar centro, Jordanésia et Polvilho. Les déplacements sont très difficiles. Jordanésia est coupé par les autoroutes Anhanguera et Bandeirantes. Les grands axes des quartiers sont toujours congestionnés. II faut faire d'énormes détours pour passer d'un site à l'autre. Jordanésia est à plus de $6 \mathrm{~km}$ de Cajamar centro tandis que Polvilho est à $8,4 \mathrm{~km}$.

\section{L'urbanisation dépendante d'une mono-activité minière}

La rencontre avec un élu passionné d'histoire, I., nous a fait comprendre que cette configuration éclatée de la ville s'explique sans doute par son histoire. En 1920, une

4 Nous avons cependant pu réaliser 10 entretiens semi-directifs avec des habitants, les uns approchés par hasard, les autres indiqués par d'autres personnes ayant participé à l'enquête, lors des visites sur le terrain. Nous avons également pu discuter avec huit leaders associatifs et politiques lors d'une réunion au Rotary Club de Cajamar. importante cimenterie de la société canadienne Ciment Portland s'installe à Perus, à côté du chemin de fer Santos-Jundiaí. Les travailleurs de la cimenterie et des mines des alentours s'établissent, eux, dans le quartier d'Água Fria, qui appartenait encore à la commune de Santana de Parnaíba et qui deviendra la municipalité de Cajamar en 1959. Le développement initial, hétéronome, de Cajamar dépendant d'une mono-activité dont le centre décisionnel se situait à l'extérieur de la future commune explique cette forte déstructuration de la ville qui s'est prolongée sous l'effet d'un développement rapide stimulé par l'implantation d'industries nouvelles. II existe donc un grand dynamisme industriel et une forte création d'emplois dans cette ville à l'urbanité embryonnaire.

\section{Le poids des occupations irrégulières dans l'urbanisation}

Les perspectives d'emploi ont attiré des migrants intérieurs nordestins (Bahia, Pernambuco, etc.) à faibles revenus qui ne peuvent se loger aux prix du marché. C'est pourquoi une part importante de la ville est constituée d'occupations irrégulières sur des terrains publics inadaptés à l'urbanisation (berges de ruisseaux, terrains en pente, etc.) et non desservis par les réseaux d'eau potable et d'assainissement. Cette situation de logement inadéquat a causé la contamination de la nappe phréatique et de l'eau de consommation.

\section{La contamination microbiologique: la conséquence cachée des occupations irrégulières}

La recherche de Maria Tereza Pepe Razzolini, de la Faculté de Santé publique de I'Université de São Paulo, notre partenaire pour la recherche exploratoire sur Cajamar, confirme l'existence du risque de contamination de l'eau dans le réseau de distribution. Les analyses indiquent une concentration dangereuse d'organismes pathogènes, Giardia et Cryptosporidium notamment, et un risque élevé d'infection. Ces protozoaires entériques peuvent se retrouver dans l'eau à la suite d'une contamination directe ou indirecte par les matières fécales d'humains ou d'animaux. Les sources de contamination sont importantes, en particulier les zones d'occupation irrégulière, dépourvues de tout système collectif d'évacuation et de traitement des eaux usées. La contamination microbiologique est un danger bien réel, mais elle n'est pas encore perçue comme une menace par la population, à la différence de l'insécurité dans les quartiers populaires, et ce, pour trois raisons. D'abord, le risque de contamination, comme la plupart des risques, est le produit d'une science mathématisée, 
technicisée, qui ne repose pas sur l'expérience entendue au sens de l'appréhension sensible de l'individu. Par conséquent, il est invisible, imperceptible, difficile à déchiffrer sans une instrumentation et une métrologie scientifique. Ensuite, les perceptions individuelles ou collectives du risque sont profondément transformées par le traitement médiatique qui le rend visible. Pour l'instant, les médias influents de Cajamar ne s'intéressent pas au problème. Enfin, les individus et les groupes sociaux sont particulièrement sensibles aux effets secondaires du risque; ici, il s'agirait des conséquences sur la santé de la contamination microbiologique. Nous n'avons pas eu le temps suffisant pour procéder à cette sensibilisation.

\section{Comment la violence et les manipulations entravent la vie publique de la cité}

Si ce développement incontrôlé de la ville, rythmé par les occupations irrégulières, a pour contrepartie la pollution de l'eau de consommation, il entraîne aussi la limitation de la démocratie par le clientélisme et l'emprise d'une économie de la drogue.

\section{Vivre dans l'insécurité d'une favela noyautée par les trafiquants}

La ville est violente. Les quartiers populaires à Jordanésia, notamment, sont noyautés par des trafiquants de drogue.

C'est un endroit dangereux, il y a beaucoup de drogue. La police ne se montre pas alors que des gens sont abattus à bout portant. Pour aller jusqu'à la mairie, je dois acquitter un soi-disant péage. Le quartier est né il y a 30 ans, d'une occupation illégale. Ils n'ont obtenu le titre de propriété des terrains que depuis deux ans. Le quartier manque de sécurité parce que la police ne vient pas ici; il manque de centres de santé, d'hôpitaux [...] Si vous publiez une partie de notre entretien, je veux qu'il soit anonymisé, car j’ai peur (J., 49 ans, Jordanésia).

Les dealers menacent l'enfant non seulement pour qu'il se drogue, mais aussi pour qu'il pousse d'autres enfants à se droguer eux-mêmes. Parfois, l'enfant est menacé ou il commence à suivre ces gens, puis ils lui demandent de l'argent et quand il ne peut pas les payer, ils lui donnent une arme blanche, un couteau... [pour qu'il se procure de l'argent en usant de la violence], ils entretiennent ainsi sa dépendance à leur égard... Les enfants deviennent même des passeurs de drogue (G., 15 ans, Jordanésia).

Malgré cela, certains habitants apprécient de pouvoir se loger à peu de frais dans une ville où ils sont susceptibles de trouver un emploi dans le secteur industriel.

\section{Une démocratie limitée par le clientélisme}

S'appuyant sur l'importance de la migration nordestine, le «clientélisme» politique s'est maintenu à Cajamar. D'après les témoignages, les politiciens élus de la ville ou leurs représentants dans le quartier aideraient les migrants récemment arrivés à Cajamar à résoudre leurs problèmes en échange de leurs votes. Ces représentants seraient généralement des professionnels dotés d'une clientèle, tels que les médecins, dentistes et pharmaciens. D’autres témoignages réfèrent à une autre modalité de clientélisme où l'influence sur les électeurs pauvres est encore plus grande. «Je suis intéressé par l'histoire. J'ai cherché à comprendre pourquoi il y avait tant de gens à Cajamar qui venaient de Buique, une ville de l'État du Pernambuco dans le Nordeste [...]. On a importé les gens de là-bas, et en échange des votes, ces gens gagnaient les terrains pour s'installer» (A., âge inconnu, Jordanésia).

Selon ces témoignages, un migrant venu du Pernambuco est devenu un notable de Cajamar. Lorsqu'il a voulu se présenter aux élections municipales, il est retourné dans la ville de Buique dont il était issu pour y chercher des électeurs. II a contacté des Nordestins pauvres à qui il a promis des avantages matériels (terrains pour s'installer, emplois, alimentation) s'ils votaient pour lui. Ils se sont donc installés à Cajamar, grossissant le nombre des occupations irrégulières, et ils ont sans doute voté pour lui. Ainsi, il existerait une corrélation entre l'apport des migrants nordestins dans la population de Cajamar, l'importance des occupations irrégulières et l'importance du clientélisme dans la vie politique.

\section{Les réseaux sociaux pour recomposer un espace public}

La rencontre avec D., propriétaire d'un bazar à Jordanésia, nous a fourni une autre vision, plus contemporaine, de la vie politique de Cajamar. Pour s'adapter à la configuration spatiale éclatée de la ville et à la violence qui limite le militantisme dans les quartiers populaires, les leaders progressistes que nous avons pu contacter, à la suite de D., se sont dotés d'un mode d'organisation original: ils animent des réseaux de développement alternatif et participatif en s'appuyant essentiellement sur les réseaux sociaux. D. utilise Facebook tandis que le Dr R., vétérinaire de Polvilho, préfère utiliser les groupes WhatsApp. Ces leaders «têtes de réseau» sont politisés, pour la plupart membres d'un parti politique (Parti des Travailleurs, Parti des Verts, etc.) et parfois de groupes religieux qui prônent l'aide aux 
plus démunis. Ils appartiennent aux couches moyennes (petit commerçant, photographe, vétérinaire, sociologue retraité, etc.) comme les représentants impliqués dans le clientélisme, mais ils revendiquent une autre conception de la politique, basée sur le débat démocratique, que ce soit sur les réseaux sociaux ou «à l'ancienne ». Ainsi, lors de notre enquête, nous avons pu assister à une réunion publique organisée par le $\operatorname{Dr}$ R. le 10 décembre 2014 au Rotary-Club de Cajamar pour sensibiliser les participants, simples habitants et leaders "têtes de réseau», aux problèmes du respect des ressources hydriques et du risque de contamination de l'eau de consommation. Ainsi, cette organisation réticulaire dote Cajamar d'un nouvel espace public animé par ces leaders où l'on débat de la vie de la cité et des problèmes environnementaux et sociaux.

\section{São Sebastião: vivre à l'ombre des cuves de Tebar}

Bien qu'elle soit située à 200 km à l'est de São Paulo, la ville de São Sebastião participe pleinement au fonctionnement de la métropole par l'importance de son terminal pétrolier, qui se situe au cœur d'une infrastructure structurante.

\section{Une ville littorale sous l'emprise du premier terminal pétrolier d'Amérique du Sud}

L'arrivée de Petrobras dans une petite ville caiçara

Cette ville de 80000 habitants est située sur le littoral nord de l'État de São Paulo. C'est une ville avec une forte identité caiçara. La culture caiçara originelle était la culture des populations traditionnelles qui habitaient entre le littoral et la Sera de Mar, la zone naturelle protégée caractérisée par une forêt primaire, la Mata Atlantica.

Selon nos interlocuteurs, ces Caiçaras étaient dotés d'une conscience écologique du lieu, leur mode de vie était économe des ressources naturelles dont ils dépendaient, car ils vivaient de pêche artisanale, d'agriculture de subsistance et de chasse. Ces communautés originelles qui possédaient les terres ont été totalement bouleversées par le développement du tourisme et des activités économiques. Une identité de résistance aux éléments les plus destructeurs (spéculation immobilière, gros équipements, etc.) s'est constituée progressivement chez leurs descendants. Elle combine la référence à l'héritage sublimé et largement mythique des Caiçaras originels et l'acceptation d'une partie de l'évolution historique.
Les nouveaux Caiçaras constituent aujourd'hui un réseau de notables. Qu'ils soient entrepreneurs, élus, artisans, artistes, journalistes ou écotouristes, ils exercent encore une forte influence sur la commune de São Sebastião. Cette influence explique peut-être pourquoi le plan directeur, le document d'urbanisme de São Sebastião, est plus protecteur des ressources naturelles (littoral et Mata Atlantica) et plus exigeant (pas d'immeubles de plus de cinq étages) que celui des municipalités voisines. Cette hypothèse s'appuie sur de nombreux témoignages recueillis lors d'une réunion publique sur les problèmes d'environnement où les participants, pour la plupart militants associatifs de protection de l'environnement, revendiquaient cette «identité caiçara» comme une justification de leur combat.

À São Sebastião, comme dans les autres villes du littoral nord, la douceur du climat, la bonne qualité générale des eaux de baignade, la présence de vagues, des vents forts et une bonne visibilité sous l'eau permettent la pratique d'activités nautiques et sous-marines pendant toute l'année. Le tourisme est favorisé. Cependant, à la différence des autres villes du littoral nord, il existe ici un facteur qui a limité le développement touristique. Depuis 1968, la ville accueille le plus grand terminal pétrolier d’Amérique latine, connu sous le nom de TEBAR, qui reçoit $50 \%$ de tout le pétrole arrivant dans le pays. Cet énorme terminal, propriété de l'entreprise Petrobras, est situé en pleine ville. Les 40 cuves immenses de TEBAR se partagent l'espace avec trois quartiers d'habitation (Vila Amelia, Porto Grande et Topolândia), le centre historique et le centre commercial de la ville. L'interpénétration spatiale de l'entreprise et de la ville est forte. Le terminal encadre la ville par ses murs et crée une véritable enclave (figure 1$)^{5}$.

L'arrivée de Petrobras a limité le développement du tourisme et l'entreprise a peut-être ainsi «protégé » l'environnement naturel qu'elle menaçait en même temps par sa pollution récurrente et la possibilité d'accidents graves. Cette situation va sans doute évoluer avec le doublement de l'autoroute Tamoios, qui relie São Sebastião et São Paulo. Pour se faire accepter, l'entreprise a contribué au développement de la ville par les emplois qu'elle offrait et l'appui qu'elle apportait aux différentes initiatives de la municipalité. Ce compromis réussi confirme les analyses de la situation de risque environnemental à São Sebastião par deux chercheurs brésiliens, une démographe et un politologue

5 Nous avons choisi à dessein cette photographie prise dans le bureau du directeur du terminal pétrolier pour illustrer l'emprise de Petrobras sur la ville de São Sebastião. 
(Santos et Marandola Jr, 2012) concernant l'importance de la confiance dans l'acceptabilité du risque, notamment celle accordée à Petrobras pour sa compétence technologique et sa contribution au développement de la zone.

FIGURE 1 Dans le bureau du directeur du terminal | Source: Lolive, 2017 | Adaptée par le Département de géographie de l'Université Laval, 2019

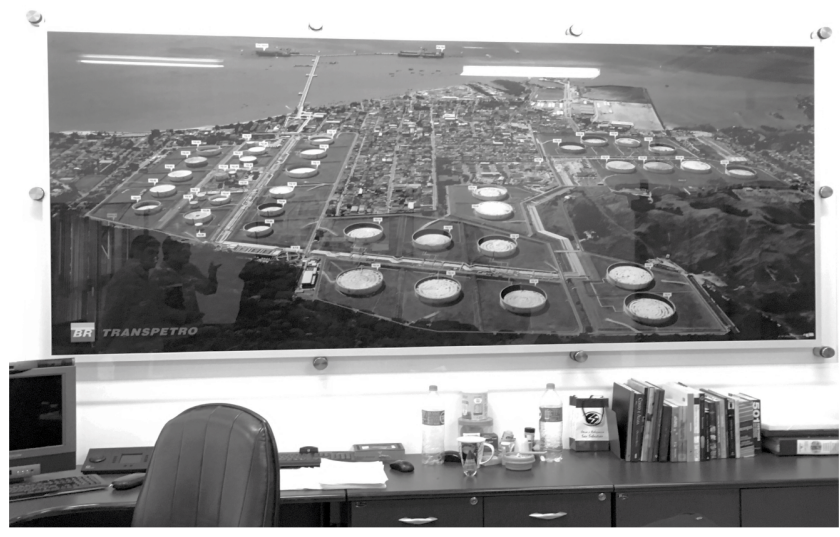

Les hypothèses catastrophiques de l'étude d'analyse de risque (EAR)

À la demande de la Companhia Ambiental do Estado de São Paulo (CETESB), l'agence gouvernementale en charge des risques pour l'État de São Paulo, par ailleurs commanditaire de notre recherche, Petrobras a commandé une EAR ${ }^{6}$. Les substances les plus dangereuses sont les dérivés «clairs» de distillation: naphte, essence, diésel et kérosène. Ces substances sont très volatiles, hautement inflammables et explosives. Ainsi, une fuite de naphte pourrait entraîner trois phénomènes dangereux: 1) si une source d'inflammation est proche, la nappe de naphte prend feu: on parle alors d'incendie de la nappe; 2) s'il ne se produit pas d'inflammation immédiate, il se forme un nuage de gaz inflammable composé d'un mélange de naphte et d'air qui peut s'enflammer à grande distance du lieu de la fuite: le flashfire est l'incendie retardé du nuage de gaz; 3) la troisième possibilité est le Unconfined Vapour Cloud Explosion (UVCE): l'incendie retardé du nuage de gaz, avec explosion.

Compte tenu de la forte interpénétration des quartiers d'habitation et du terminal, l'hypothèse accidentelle la plus grave pourrait causer jusqu'à 200 décès. C'est une hypothèse catastrophique dans la mesure où la

6 Cette EAR répond à la norme technique CETESB P4.261 concernant les risques d'accidents technologiques. Elle permet à la CETESB d'exercer un contrôle sur les entreprises à haut risque. Elle n'a rien à voir avec l'analyse de la situation du risque environnemental à São Sebastião dont nous avons parlé précédemment. catastrophe est un événement - ou plus souvent une combinaison d'événements - brutal ou durable et intense, d'origine naturelle ou humaine, entraînant généralement la mort et des destructions à grande échelle. La catastrophe technologique est due en partie à une série de défaillances technologiques et humaines dans une entreprise dangereuse pour l'environnement, comme Petrobras.

\section{La catastrophe, un point aveugle qui influence pourtant les comportements}

Le risque influence la vie collective à São Sebastião d'une manière spécifique, car il ne s'agit pas d'un risque ordinaire mais de la possibilité d'une véritable catastrophe. Cette perspective est difficilement supportable, elle constitue une sorte de point aveugle, ce dont témoigne l'importance des stratégies de déni du risque chez les administrations et les habitants les plus menacés.

\section{Le déni du risque à Vila Amelia: quand les fleurs masquent les cuves}

Pour comprendre comment cette situation était vécue au quotidien par les habitants, nous avons étudié le quartier le plus enclavé, celui de Vila Amelia, qui est profondément inséré dans le site du terminal. Les murs de Petrobras encadrent ce quartier et les immenses cuves de TEBAR dominent les petites maisons individuelles de Vila Amelia. «Un plan d'évacuation des gens serait difficile pour les habitants de Vila Amelia qui sont enclavés. En cas de catastrophe, ils seront pris au piège et ne pourront pas sortir!» (S., âge inconnu, habitant de Vila Amelia). La vulnérabilité est encore accrue par le grand nombre d'équipements collectifs: on y trouve notamment trois écoles, un gymnase, un collège, une université, un poste de police locale et la clinique de São Sebastião.

Ce qui frappe, dans le quartier Vila Amelia, c'est l'omniprésence des fleurs, hibiscus, bougainvillées, arbustes avec des belles fleurs jaunes, palmiers qui dominent de petits jardins exubérants. C'est un quartier résidentiel composé de coquettes petites maisons de plain-pied aux couleurs pastel avec des petits commerces de service (immobilier, informatique, salon de beauté), un quartier populaire, vivant et accueillant. L'entreprise est comme un arrière-plan menaçant partout présent, mais qu'on ne perçoit pas. TEBAR n'est visible que dans les encoignures. Parfois, une partie de cuve apparaît cachée derrière la chapelle ou au fond d'une ruelle qui longe une maison. Dans une parcelle sans habitation, le mur de TEBAR apparaît, environ $5 \mathrm{~m}$ de haut avec une frise de 
barbelé, gris, sale et très laid, mais la mairie y a installé un petit square minuscule avec des arbres et des fleurs, un banc, et une balançoire pour les enfants. Tout au fond, le haut mur gris, plutôt sinistre, barre la rue avec, derrière, une cuve immense. Les haut-parleurs sur le mur évoquent le danger plus encore que les cuves. Un message en grosses lettres est inscrit sur le mur: «EN CAS D'URGENCE APPELER LE 0800-128 121». Le déni du risque, ce sont ces deux visions qui se télescopent: les petites maisons fleuries qui masquent l'entreprise et le grand mur laid de TEBAR qu'il est difficile d'ignorer avec ses haut-parleurs, ses messages d'urgence et les immenses cuves derrière lui. Un témoignage pour illustrer ce déni: le directeur d'une des écoles de Vila Amelia «ne sait pas à quoi servent les sirènes ». Elles sont pourtant juste en face de l'école!

Cette attitude de déni du risque a été étudiée dans l'article de l'anthropologue Françoise Zonabend (1993), qui analyse les stratégies défensives mises au point par les employés et les riverains de l'usine de la Haque en Cotentin (France) pour travailler et vivre sans trop de souffrances auprès de ces établissements à haut risque qui suscitent l'anxiété. Selon elle, «une posture de déni permet d'apprivoiser cet univers dangereux et inconnu dans lequel il faut vivre et demeurer»».

Pour vivre avec le nucléaire, il faut l'oublier, et pour ce faire il n'est pas de meilleur moyen que de n'en pas parler, de ne pas nommer ces usines inquiétantes. Les riverains de l'usine de retraitement des combustibles irradiés ne la nomment pas. Quand ils en parlent, ils disent «l'usine», sans plus de qualification, mais le plus souvent ils disent seulement «là-haut», ou encore «la chose», ou «ça», toutes façons de dire qui impliquent, on le sent bien, un désir d'éloignement, une mise à distance. Et ce lieu non nommé, ils tentent par toutes sortes de moyens de ne pas le voir. "L'usine, de chez moi, on ne la voit pas... Alors on est protégé », m'assure un habitant d'un hameau situé à quelques centaines de mètres à vol d'oiseau de l'établissement. Pourtant, sortant de chez lui, il m'a suffi de regarder vers l'arrière de sa maison pour contempler, immense et présente, l'usine plantée là au bout de sa cour (Zonabend, 1993: 129).

\section{Le souvenir d'un accident grave qui préfigurait} la catastrophe

Même s'il ne s'est pas produit jusqu'alors de véritable catastrophe à São Sebastião, un événement grave a fortement marqué les esprits parce qu'il a constitué une véritable préfiguration de la potentialité d'une catastrophe: l'incendie du Córrego de Outeiro. Le «ruisseau de la colline» traverse les installations de TEBAR, le quartier de Vila Amelia et le centre-ville. Le 6 avril 1984, la fuite du pétrole d'une cuve de stockage se déverse dans le Córrego. La nappe prend feu et des flammes de $10 \mathrm{~m}$ de haut voyagent avec le ruisseau à travers le centre-ville, menaçant un hôpital et de nombreux services et habitations avant de se jeter dans le canal de São Sebastião, y causant une marée noire (Platon, 2010). L'événement provoque une panique généralisée de la population et le blocage des routes. Il laisse la ville sans électricité, sans téléphone et sans eau. Fort heureusement, il n'y a qu'un mort et le pire est évité. Cet accident grave renforce la plausibilité de l'événement catastrophique.

\section{L'insuffisante mobilisation des institutions}

Inquiétées par cette possibilité d'un accident catastrophique et contraintes par notre travail de sensibilisation des habitants, les institutions concernées (mairie, défense civile, pompiers, entreprise Petrobras, CETESB) ont décidé de mettre en œuvre l'Awareness and Preparedness for Emergencies at Local Level (APELL), une procédure de sensibilisation et préparation aux situations d'urgence au niveau local préconisée par les Nations Unies, mais elles sont réticentes à impliquer de manière active les habitants dans la procédure ${ }^{7}$. Ceux-ci n'y apparaissent qu'en tant que population passive qu'il faut déplacer pour l'éloigner du danger. Nous sommes en train de changer cette conception restrictive en encourageant la constitution d'un forum des habitants, qui deviendra l'interlocuteur du comité de réalisation d'APELL.

\section{Faire l'expérience sensible et imaginaire d'une catastrophe pour mieux s'y préparer}

\section{Comment la catastrophe pourrait métamorphoser le milieu de vie}

Le Córrego de Outeiro apparaît comme un véritable trait d'union entre TEBAR, le quartier d'habitation de Vila Amelia, le centre-ville et la plage avec les pêcheurs. La municipalité a réalisé des aménagements paysagers tout le long de son parcours. Le Córrego actuel est paisible, accueillant, tout comme le quartier de Vila Amelia s'efforce de l'être. Un événement catastrophique transformerait radicalement ce milieu de vie à l'image de la préfiguration de 1984. Le petit ruisseau familier deviendrait une rivière

7 Outre l'expérience du théâtre-forum qui sera présentée plus loin, nous organisons dès cette année une formation aux risques technologiques de 30 heures annuelles s'appuyant sur l'exemple de São Sebastião et destinée aux enseignants de deux écoles de la zone à risque. 
de feu et le petit quartier paisible deviendrait un enfer. Ainsi, cette capacité de «métamorphose» du milieu de vie constitue une des caractéristiques permettant de distinguer l'événement catastrophique de la réalisation du risque. Elle nous impose de définir une stratégie spécifique de sensibilisation préalable d'une population exposée à la catastrophe. Cette stratégie est illustrée par l'expérience du théâtre-forum.

\section{L'expérience du théâtre-forum}

Pour les riverains, la catastrophe est plus difficile à imaginer que l'accident ordinaire, car elle marque une véritable rupture avec la vie de tous les jours et métamorphose les lieux du quotidien. C'est pourquoi, en accord avec l'entreprise, nous avons transposé la méthode du théâtreforum, créée par le dramaturge brésilien Augusto Boal, pour construire une situation dans laquelle les habitants du quartier vont faire l'expérience sensible et imaginaire d'une catastrophe simulée. Avec l'aide de l'entreprise et des institutions concernées, nous avons défini un scénario de risque: un incendie se déclare dans une cuve de pétrole à l'intérieur du terminal. Ce scénario est mis en scène par un réalisateur et interprété par des acteurs qui interpellent le public, composé des habitants et des instituions gestionnaires du risque, pour qu'il participe à un débat. Cinq scènes représentent différentes situations: 1 ) un fils essaie de lever sa mère âgée et alitée pour se conformer à la demande d'évacuer le quartier; 2) la professeure d'une école située à Vila Amélia ne sait pas comment évacuer les élèves de l'école et où aller; 3 ) les voitures sont bloquées par un énorme embouteillage; un camion chargé d'un produit pour étouffer l'incendie tente d'arriver à TEBAR, mais les voitures lui barrent la route; 4 ) une mère a perdu son enfant qui était sous la responsabilité de l'école; et 5) une professeure ignore quelles substances sont stockées dans les cuves du terminal et ne sait pas ce qui pourrait arriver si une cuve prend feu.

Cette simulation rendue « impliquante », grâce aux savoirfaire artistiques, a permis que les institutions prennent conscience de leur manque de préparation pour faire face aux situations mises en scène. Elle a aussi suscité un fort désir des habitants qui participaient d'être associés à notre démarche. Notre proposition de constituer un forum des habitants a été bien reçue. Ainsi, le public a vécu par l'imagination cette expérience d'une catastrophe qui permet de mieux se préparer à l'événement et d'améliorer les dispositifs existants. La sensibilisation des habitants de São Sebastião devrait s'appuyer sur ce type d'expérience et sur l'organisation des écoles comme centres de communication du risque en direction des habitants.

\section{Condominío Barão de Mauá: habiter une zone contaminée}

Le troisième exemple nous permettra d'illustrer l'importance de la contamination des sols par l'industrie dans une métropole où l'on compte à ce jour 3181 zones contaminées. Elles sont l'héritage de l'industrialisation.

Dans les années 80, une déconcentration industrielle s'est opérée dans les zones les plus urbanisées du Brésil. Ce phénomène a contribué à l'apparition de nouvelles zones de risque, suite au changement de l'usage des sols urbains. Des habitations et des services ont été construits sur ces zones sans que l'on ait réalisé des études spécifiques de milieu pour des récupérations ultérieures et/ou un assainissement du sol (Ramires et Ribeiro, 2011).

Comment habiter le Condominío Barão de Mauá, un ensemble résidentiel situé dans la ville de Mauá, à $30 \mathrm{~km}$ de São Paulo? Les bâtiments ont été construits sur une zone contaminée. Une procédure judiciaire est en cours, mais elle s'éternise et, depuis 18 ans, les résidents sont exposés au double risque de contamination par des substances cancérigènes (le benzène et ses dérivés) et d'explosion par le méthane. Un travail d'enquête réalisé au Barão de Mauá en juin 2015 s'est appuyé sur un temps fort, une immersion anthropologique de cinq jours (du 1er au 5 juin 2015) durant laquelle l'équipe de recherche franco-brésilienne a habité dans le condominium pour mieux comprendre la manière dont la zone contaminée était habitée et vécue par ses habitants. Cette approche donnait accès à l'expérience sensible quotidienne des résidents, à la manière dont un territoire à risque peut être vécu de l'intérieur et mobiliser ses habitants.

\section{Comment la contamination dégrade le milieu de vie et affecte ses habitants}

\section{Le traumatisme de l'explosion meurtrière}

Le 20 avril 2000, une explosion se produit dans le condominium, tuant un ouvrier et en brûlant grièvement un autre. Cet événement dramatique cause un traumatisme réel, surtout pour ceux qui ont assisté à la scène. Les habitants vivent désormais dans la crainte d'une explosion. Lors des débats collectifs, leurs plaintes constituent l'expression publique d'une vulnérabilité. Les habitants évoquent leurs souffrances: le traumatisme de «ceux qui 
ont vu le gars brûlé, nu, sans tête», «le rêve d'habiter ici qui tourne au cauchemar», la terreur de vivre ici «avec ces monstres qui semblent prêts à exploser à tout moment», la stigmatisation dont ils sont l'objet. Ils rappellent les maladies liées à la contamination, les enfants qui ont peutêtre le cancer, les voisins malades qui s'en vont, les tests épidémiologiques aux résultats ambigus et le caractère diffus de la contamination.

\section{La mise en suspens de toute évolution du condominium}

L'explosion inaugure la mise en suspens de toute l'évolution du condominium. Les immeubles inachevés, des bâtiments fantômes sans portes ni fenêtres, dont la construction s'est arrêtée le jour de l'explosion, en témoignent (figure 2).

FIGURE 2 Les immeubles fantômes | Source: Lolive, 2017 | Adaptée par le Département de géographie de l'Université Laval, 2019

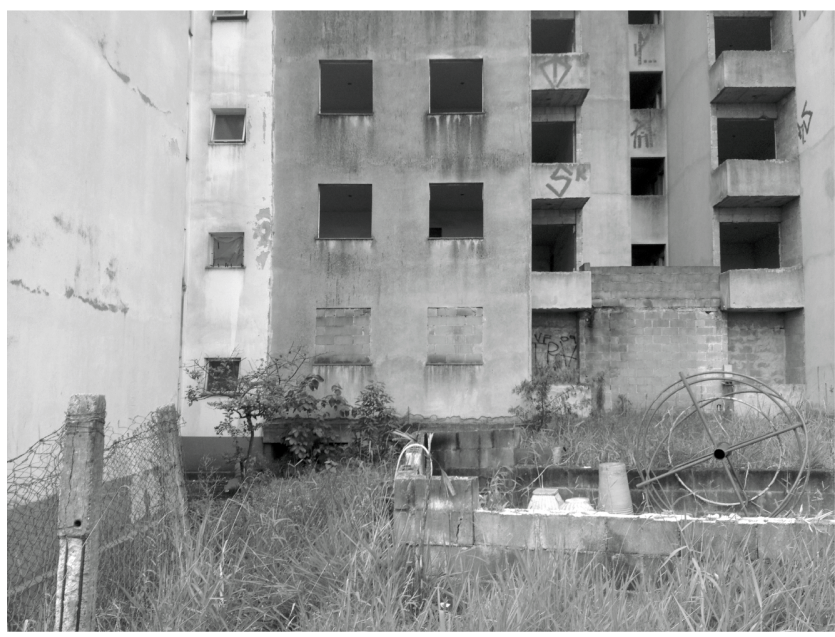

Le futur semble suspendu. La plupart des habitants attendent les résultats de décisions juridiques fixant le montant de compensations possibles ou les résultats d'expertises: la révélation d'un niveau de toxicité nécessitant leur départ ou un possible résultat d'analyses épidémiologiques prouvant une contamination par le benzène. Cette situation rend difficile les décisions normales de toute trajectoire de vie (partir, louer, vendre, acheter, rénover). En attendant, il n'y a pas de projet possible pour l'évolution du quartier: aucune salle commune, pourtant désirée, n'est construite, car toute nouvelle fondation est risquée. Impossible de lancer la moindre rénovation des bâtiments, et même de démolir ou de transformer ce qui a été arrêté et depuis longtemps abandonné.

\section{La contamination altère les relations des habitants avec leur milieu de vie}

Les témoignages des habitants traduisent une perte de confiance aux repères sensibles. L'odorat, la vue ne sont plus fiables, les sens des habitants sont abusés. Malgré leur belle apparence, on ne peut pas manger les fruits sur les arbres du condominium. Ils sont réservés aux oiseaux. Les entretiens témoignent d'une vigilance accrue. Une question lancinante s'exprime dans les propos des habitants: le méthane, le benzène ont-t-ils une odeur? Au niveau sonore, de nombreux habitants gardent en mémoire l'explosion de 2000 , si bien que, lorsqu'ils entendent un bruit de choc soudain, ils ne peuvent s'empêcher de penser à l'éventualité d'une autre explosion. Les habitants doivent apprendre à vivre avec le risque dans le condominium et la vigilance traduit peut-être une tentative de retrouver la confiance dans le sensible en renouant des liens avec un environnement devenu inhospitalier.

\section{Restituer le travail multiforme des habitants pour rendre habitable la zone contaminée}

Cependant, une aire contaminée est aussi un milieu de vie. Les entretiens restituent l'effort pour vivre dans cette zone contaminée et l'énergie de la vie quotidienne qui continue.

\section{Habiter le condominium en dépit du risque}

La volonté d'habiter là en dépit du risque s'exprime avec force: «le condominium est bien, il est tranquille, sécuritaire: Je vous dis que j'aime beaucoup mon appartement. Quand les gens disent «ah, vous habitez à Tchernobyl» dans la zone contaminée, non! j’habite dans mon appartement. J'habite chez moi. Compris? Je n'habite pas à Tchernobyl. J'habite dans ma maison. Ma maison, je ne la considère pas comme une poubelle. Je la considère comme une maison » (C., âge inconnu, concierge et habitante du condominium). La majorité des résidents préfèrent rester dans le condominium plutôt que d'habiter à Jardim Zaíra (un quartier populaire de Mauá).

\section{La solidarité qui s'est développée entre les habitants}

La persistance du milieu de vie s'exprime également à travers la solidarité qui s'est développée entre les habitants. Si le condominium n'a pas d'espace collectif, se sont instaurés des lieux de rencontres, de débats et d'échanges: les stationnements, l'espace autour des portails d'entrée, les loges des gardiens et les halls des immeubles. Cette solidarité s'est exprimée dans la forte 
mobilisation des habitants pour obtenir une réparation de la part des entreprises qui ont contaminé le terrain, construit les immeubles et commercialisé les logements en toute connaissance de cause. Malgré sa durée d'une dizaine d'années environ, cette mobilisation n'a pas eu les résultats escomptés.

\section{Le home sweet home: un fragile rempart esthétique contre la contamination}

Cette tentative de conjurer la peur, les efforts pour vivre dans cette zone contaminée se traduisent notamment par la production d'un home sweet home selon l'expression de Nicolas Tixier, chercheur au Centre de recherche sur l'espace Sonore et l'environnement urbain (CRESSON) et membre de notre équipe. En témoignent les pelouses impeccablement tondues en bas des bâtiments, les bancs blancs en fer forgé qui les décorent, sans oublier les petits animaux en plâtre inspirés de Walt Disney et les petits panneaux aux couleurs pastel avec des messages lénifiants comme «Dieu bénit cette maison» ou «Le bonheur habite ici». Cet aménagement exprime le triomphe d'une esthétique kitsch.

La zone contaminée menace le milieu de vie des résidents, à la fois fragilisé par la contamination chimique et partiellement approprié par les ingénieurs de la CETESB et de l'entreprise de décontamination Geoklock, qui le considèrent comme un simple champ d'expérimentation biophysique. Ainsi, les techniciens de Geoklock sont partout dans le condominium avec leurs combinaisons, leurs outils, leur matériel. Mais il semble que, pour eux, les habitants soient transparents; ils ne leur parlent pas et ne répondent pas aux questions, comme si ces résidents n'existaient pas. À part les réunions obligatoires d'information, ils n'associaient pas les habitants à la décontamination (mais il semble que la situation se soit améliorée depuis). La production du home sweet home vise à recréer dans la zone contaminée un lieu aménagé et approprié qui prolonge le logement et protège symboliquement les habitants. Les caractéristiques de ce lieu humanisé, chaleureux, entretenu, saturé de sens, empreint de sentimentalité jusqu'au ridicule, s'opposent en effet point par point à celles de l'espace contaminé, désolé, hostile, dangereux et dépourvu de sens qu'il s'agit de tenir à distance ${ }^{8}$. Cette petite bulle protectrice de bibelots et de mots offre un

8 La relative perte de sens pour les habitants de ce milieu de vie contaminé, perceptible dans les entretiens, pourrait s'expliquer par le fait que la partie contaminée de leur milieu de vie leur est en quelque sorte soustraite, dans la mesure où elle est «objectivée » et appropriée par les techniciens de Geoklock et de la CETESB. fragile rempart sensible, esthétique et symbolique à la contamination du milieu de vie.

\section{La participation à la gestion du risque pour rétablir la confiance des habitants}

Pour commencer à améliorer la situation, nous avons proposé de mettre en œuvre une gestion participative et partenariale du risque à Barão de Mauá basée sur un nouveau dispositif de participation composé de deux forums complémentaires appelés à travailler ensemble, l'un comprenant des habitants du condominium et l'autre des différentes institutions concernées. Les réunions avec les habitants ont permis de mettre en évidence un enjeu essentiel que nous sommes en train de traiter. Cet enjeu concerne la confiance perdue à l'égard des institutions.

Lors des premières réunions, en effet, la question du risque a semblé moins importante que celle de la confiance accordée aux organisations qui le prennent en charge, notamment la CETESB. À notre grande surprise, les habitants n'ont pas formulé de propositions concernant l'amélioration de leurs conditions de vie dans le condominium. Ils ont d'abord exprimé une demande très forte d'avoir une réunion avec les institutions pour qu'elles leur présentent en détail le projet de décontamination du site et l'évaluation qu'en avait faite la CETESB. C'était une sorte de préalable pour juger de notre bonne foi. Ils ont insisté pour que leur interlocuteur de la CETESB ait un «langage de vérité ». Un dialogue difficile s'est instauré lors de la première rencontre. Le processus de gestion partagée de la contamination et du dispositif de dépollution du Condominío Barão de Mauá, que nous sommes en train d'expérimenter pour que les habitants puissent influencer un dispositif de gestion et de dépollution essentiellement technocratique, pourrait permettre de restaurer la confiance des habitants, s'il n'est pas trop tard, car leur situation d'habitants lourdement exposés dure depuis bien trop longtemps maintenant.

\section{Quelques résultats de recherche}

Pour conclure, nous allons discuter les résultats obtenus par notre recherche en revenant sur les questions principales qui l'ont balisée: comment analyser la diversité des situations de risque dans la RMSP? L'utilisation de la notion de monde social du risque pour analyser trois situations de risque nous a permis de mettre en évidence les différentes caractéristiques de chacun des mondes du risque que nous rappelons ici (tableau 1) 
Malgré l'intérêt des analyses qu'elle permet, la notion de monde du risque doit être améliorée pour rendre compte du fonctionnement du monde du risque. Pour mieux le comprendre, il reste à analyser la dimension systémique de ce monde à travers la transaction entre cet organisme social et ses environnements, c'est-à-dire le rapport entre les relations internes et les relations externes du monde social du risque. Cette analyse fournirait un diagnostic concernant la situation que l'émergence d'une culture du risque pourrait modifier. Le tableau qui suit ébauche quelques pistes en la matière (tableau 2).

TABLEAU 1 Trois mondes sociaux du risque en cours de constition dans la métropole de São Paulo | Conception: Lolive et Okamura, 2018

\begin{tabular}{|c|c|c|c|c|}
\hline & $\begin{array}{c}\text { Constitution } \\
\text { d'un milieu de vie } \\
\text { transformé par le risque }\end{array}$ & $\begin{array}{l}\text { Principaux risques } \\
\text { concernés }\end{array}$ & $\begin{array}{l}\text { Le risque transforme le champ } \\
\text { des activités collectives }\end{array}$ & $\begin{array}{c}\text { Émergence d'une culture du } \\
\text { risque par la publicisation } \\
\text { du risque }\end{array}$ \\
\hline Cajamar & $\begin{array}{l}\text { Une ville éclatée dont la } \\
\text { majorité des quartiers } \\
\text { d’habitation sont } \\
\text { constitués de favelas }\end{array}$ & $\begin{array}{c}\text { Violence liée au trafic } \\
\text { de drogue. } \\
\text { Contamination } \\
\text { microbiologique des eaux }\end{array}$ & $\begin{array}{l}\text { Les trafiquants noyautent } \\
\qquad \text { la favela. } \\
\text { Vie politique marquée } \\
\text { par le clientélisme }\end{array}$ & $\begin{array}{l}\text { Les leaders progressistes } \\
\text { utilisent les réseaux sociaux } \\
\text { pour recomposer un espace } \\
\text { public }\end{array}$ \\
\hline São Sebastião & $\begin{array}{l}\text { Une ville avec ses } \\
\text { quartiers d'habitation } \\
\text { imbriqués dans un } \\
\text { terminal pétrolier }\end{array}$ & $\begin{array}{l}\text { Hypothèse catastrophique: } \\
\text { incendie et explosion d'un } \\
\text { nuage de gaz: } 200 \text { morts }\end{array}$ & $\begin{array}{l}\text { La perspective d'une } \\
\text { catastrophe alimente le déni } \\
\text { du risque très courant chez les } \\
\text { habitants et les gestionnaires }\end{array}$ & $\begin{array}{l}\text { L'expérience sensible } \\
\text { et imaginaire d'une } \\
\text { catastrophe pour mieux } \\
\text { s'y préparer }\end{array}$ \\
\hline $\begin{array}{l}\text { Condominío } \\
\text { Barão de } \\
\text { Mauá }\end{array}$ & $\begin{array}{c}\text { Un ensemble résidentiel } \\
\text { construit sur une zone } \\
\text { contaminée }\end{array}$ & $\begin{array}{l}\text { Contamination par des } \\
\text { substances cancérigènes. } \\
\text { Explosion par le méthane }\end{array}$ & $\begin{array}{l}\text { La contamination dégrade le } \\
\text { milieu de vie de ses habitants, } \\
\text { ce qui suscite l'expression des } \\
\text { affects au sein de } \\
\text { la population. } \\
\text { Le travail multiforme des } \\
\text { habitants pour rendre } \\
\text { habitable la zone contaminée }\end{array}$ & $\begin{array}{l}\text { Après l'échec d'une } \\
\text { première mobilisation, un } \\
\text { processus participatif pour } \\
\text { associer les habitants à la } \\
\text { gestion du risque } \\
\text { de contamination }\end{array}$ \\
\hline
\end{tabular}

TABLEAU 2 Fonctionnement des trois mondes sociaux du risque considérés comme systèmes (ébauche) | Conception: Lolive et 0kamura, 2018

\begin{tabular}{|c|c|c|c|}
\hline & $\begin{array}{c}\text { Relations internes: } \\
\text { degré d'intégration du monde du risque }\end{array}$ & Relations externes & $\begin{array}{l}\text { Diagnostic } \\
\text { concernant le monde } \\
\text { du risque considéré } \\
\text { comme un système }\end{array}$ \\
\hline Cajamar & $\begin{array}{c}\text { Absence d'intégration: atomisation } \\
\text { du collectif par violence, manipulations et } \\
\text { absence d'urbanité }\end{array}$ & $\begin{array}{l}\text { Ouverture aux logiques économiques et aux } \\
\text { migrations externes }\end{array}$ & Hétéronomie \\
\hline São Sebastião & $\begin{array}{l}\text { Difficile intégration autour d'un intérêt mal } \\
\text { perçu (la catastrophe) malgré une sensibilité } \\
\text { environnementale qui s'appuie sur l'héritage } \\
\text { sublimé de la culture caiçara }\end{array}$ & $\begin{array}{c}\text { Ouverture au tourisme limitée par la } \\
\text { présence de Petrobras mais qui va se } \\
\text { développer avec le doublement de } \\
\text { l'autoroute }\end{array}$ & Équilibre menacé \\
\hline $\begin{array}{l}\text { Condominío } \\
\text { Barão de Mauá }\end{array}$ & $\begin{array}{c}\text { Forte intégration (solidarité) autour d'une } \\
\text { situation commune (contamination) et } \\
\text { d'une uniformité sociale (couches sociales } \\
\text { moyennes basses) }\end{array}$ & $\begin{array}{l}\text { Fermeture relative vis-à-vis des quartiers } \\
\text { voisins (condominium fermé dont les } \\
\text { habitants sont stigmatisés). } \\
\text { Repli sur soi après une mobilisation longue et } \\
\text { sans résultat évident }\end{array}$ & Relatif isolement \\
\hline
\end{tabular}


Pour ce qui est de notre seconde question: comment vivre avec le risque?, notre recherche nous révèle que l'expérience du risque suscite un apprentissage sensible, social et politique de la population. Ainsi, les habitants doivent expérimenter de nouvelles pratiques pour renouer des liens avec un environnement devenu inhospitalier. L'habiter, qui repose sur l'expérience des habitants, devient expérimental dans les mondes du risque. Cette recherche nous a rendus attentifs aux expériences d'adaptation créative des habitants dont le home sweet home du Condominío Barão de Mauá, le déni du risque à São Sebastião et l'usage des réseaux sociaux à Cajamar. Même si cela nécessiterait une étude approfondie pour le démontrer, notre approche basée sur l'enquête de sens commun (Dewey, 1993) des habitants semble élargir la gamme des ajustements possibles au sein des groupes sociaux au regard de leur perception et de leur prévention des risques dans l'approche classique des géographes américains (Burton et al., 1978) qui les analysaient comme réponses adaptatives aux risques naturels.

Nous avons choisi une posture de recherche pragmatique qui justifie notre implication dans le processus de constitution des mondes du risque que nous étudions. Cela nous a conduits, notamment, à analyser et à favoriser la publicisation de ce processus essentiel qui confère au monde du risque sa réflexivité. Cette posture originale doit être évaluée à la fois sur ses résultats théoriques et sur les effets pratiques qu'elle est susceptible de produire à l'issue d'une intervention limitée sur chacun des terrains ${ }^{9}$. C'est ce qui la rend difficile à exercer. Sur Cajamar, notre recherche était exploratoire et si nous avons pu repérer la constitution d'un public par les réseaux sociaux, nous n'avons pas eu le temps de soutenir cette constitution. Sur le Condominío Barão de Mauá, le public était déjà constitué depuis l'explosion de 2001, mais il n'est pas parvenu à influencer suffisamment les institutions. L'échec de sa mobilisation et l'inertie des institutions lui fait traverser actuellement une phase de découragement. Si les représentants des habitants participent à notre forum participatif, il subsiste une grande méfiance à l'égard des institutions. Sur São Sebastião, le public n'est pas encore constitué autour des enjeux de la catastrophe technologique et notre travail consiste à sensibiliser à la fois les habitants et les institutions, c'est-àdire à créer les conditions pour qu'ils se sentent concernés. C'est pourquoi la situation est plus favorable.

9 La recherche dont il est question ici s'est terminée en mars 2019. Elle aura duré quatre ans en tout, soit deux années pour la recherche proprement dite et deux années pour l'opérationnalisation des résultats.
Pour apprécier vraiment l'importance du pragmatisme dans notre travail, il faut dépasser la question de l'engagement des chercheurs dans le processus de publicisation et présenter le programme de recherche qui a inspiré cet article consacré à l'analyse des mondes sociaux. Notre recherche présentait une dimension opérationnelle qui n'a pas fait ici l'objet d'une présentation détaillée. Elle visait en effet à expérimenter différentes méthodes qualitatives pour tester la participation des habitants dans la communication et la gestion du risque avec les populations exposées de la RMSP. Nous sommes en train d'opérationnaliser les connaissances produites par cette recherche pour en tirer une norme réglementaire de préparation des communautés exposées aux situations de risque technologique, laquelle sera mise en œuvre par notre partenaire institutionnel, la CETESB, l'agence gouvernementale en charge de la gestion des risques et de l'environnement. Ces protocoles modifieront l'action des administrations partenaires et des entreprises, ils transformeront les politiques de gestion des risques dans l'État de São Paulo pour les ouvrir aux diagnostics des sciences humaines et à la participation des habitants. Ainsi, nous aurons atteint l'objectif central de notre projet: réaliser une recherche en sciences sociales qui puisse transformer les politiques publiques.

\section{Bibliographie}

BECK, Ulrich (2001) La société du risque. Sur la voie d'une autre modernité. Paris, Aubier.

BECK, Ulrich, GIDDENS, Antony et LASH, Scot (1994) Reflexive modernization. Politics, tradition and aesthetics in the modern social order. Cambridge, Polity Press.

BECKER, Howard (1976) Art worlds and social types. American Behavioral Scientist, vol. 19, nº6, p. 703-718.

BERQUE, Augustin (2000) Écoumène. Introduction à l'étude des milieux humains. Paris, Belin

BOURDIN, Alain (2003) La modernité du risque. Cahiers internationaux de sociologie, vol. 1, n¹14, p. 5-26.

BRITISH STANDARD OCCUPATIONAL HEALTH AND SAFETY ASSESSMENT SERIES (BS OHSAS) (2007) Norme internationale de management de la santé et de la sécurité au travail par les entreprises, révisée en juillet 2007. Genève, Organisation internationale du Travail.

BRUNET Philippe (2008) De l'usage raisonné de la notion de «concernement»: mobilisations locales à propos de l'industrie nucléaire. Natures Sciences Sociétés, vol. 16, n4, p. 317-325. 
BURTON, Ian, KATES, Robert William et WHITE, Gilbert F. (1978) The environment as hazard. New York, Oxford Press University.

CANEVACCI, Massimo (1997) A cidade polifônica. São Paulo, Studio Nobel.

CEFAÏ, Daniel (2013) Public, socialisation et politisation: Mead et Dewey. Dans Alexis Cukier et Éva Debray (dir.) La théorie sociale de George Herbert Mead. Paris, La Découverte. p. 340-364.

CEFAÏ, Daniel (2015) Mondes sociaux. Enquête sur un héritage de l'écologie humain à Chicago. SociologieS. [En ligne]. http://journals.openedition.org/sociologies/4921

CEFAÏ, Daniel, BIDET, Alexandra, STAVO-DEBAUGE, Joan, FREGA, Roberto, HENNION, Antoine et al. (2015) Introduction du Dossier «Pragmatisme et sciences sociales: explorations, enquêtes, expérimentations». SociologieS [En ligne]. http://journals.openedition. org/sociologies/4915

COMPANHIA AMBIENTAL DO ESTADO DE SÃO PAULO (CETESB) (2017) Relação de áreas contaminadas. São Paulo, CETESB.

DARDEL, Éric (1990) L'Homme et la Terre: nature de la réalité géographique. Paris, Éditions du Comité des travaux historiques et scientifiques.

DAYNAC, Michel (2002) Pragmatisme, expertise et énoncés scientifiques. Réflexions sur l'utilisation de l'expertise dans la formulation des énoncés scientifiques en sciences sociales. Paris, Association française de Science des Systèmes [En ligne]. http://www.afscet.asso.fr/ resSystemica/Crete02/Daynac.pdf

DEWEY, John (1993) Logique. La théorie de l'enquête. Paris, Presses universitaires de France.

DEWEY, John (2010) Le public et ses problèmes. Paris, Gallimard, [1927].

DOUGLAS, Mary (1999) Four cultures: The evolution of a parsimonious model. GeoJournal, vol. 47, n³, p. 411-415.

DOUGLAS, Mary et WILDAVSKY, Aaron (1983) Risk and culture. An essay on the selection of technological and environmental dangers. Berkeley, University of California Press.

GIRARD, Tobias (2013) Comment pense Mary Douglas? Risque, culture et pouvoir. Ethnologie française, vol. 43, n¹, p. 137-145.

GUSFIELD, Joseph R. (2007) La culture des problèmes publics. L'alcool au volant: la production d'un ordre symbolique. Paris, Economica.
HENNION, Antoine (2015) Enquêter sur nos attachements. Comment hériter de William James? SociologieS [En ligne]. https://journals.openedition.org/ sociologies/4953

HOYAUX, André-Frédéric (2002) Entre construction territoriale et constitution ontologique de l'habitant: introduction épistémologique aux apports de la phénoménologie au concept d'habiter. Cybergeo: revue européenne de géographie [En ligne]. http://journals.openedition. org/cybergeo/1824

HOYAUX, André-Frédéric (2015) Habiter: se placer plaçant et se penser pensant. Annales de géographie, vol. 704, $n^{\circ} 4$, p. 366-384.

INSTITUTO BRASILEIRO DE GEOGRAFIA E ESTATÍSTICA (IBGE) (2011) Censo demográfico 2010. Aglomerados subnormais. Primeiros resultados. Rio de Janeiro, Ministério do Planejamento, Orçamento e Gestão.

INSTITUTO BRASILEIRO DE GEOGRAFIA E ESTATÍSTICA (IBGE) (2018) Estimativas da população residente nos municípios brasileiros com data referência em 10 de julho de 2018. Rio de Janeiro, Ministério do Planejamento, Orçamento e Gestão.

JAMES, William (2005) Essais d'empirisme radical. Marseille, Agone.

KASPERSON, Roger E., RENN, Ortwin, SLOVE, Paul, BROWN, Halina S., EMEL, Jacque et al. (1988) The social amplification of risk: A conceptual framework. Risk Analysis, vol. 8, n², p. 177-187.

LAPOUJADE, David (1997) William James. Empirisme et pragmatisme. Paris, Presses universitaires de France.

LATOUR, Bruno (1991) Nous n'avons jamais été modernes. Essai d’anthropologie symétrique. Paris, La Découverte.

LE BOTERF Guy (1983) La recherche-action: une nouvelle relation entre les experts et les acteurs sociaux? Pour, n॰90, p. 39-46.

LEWIN, Kurt (1946) Action research and minority problems. Journal of Social Issues, vol. 2, n4, p. 34-36.

MARTINAIS, Emmanuel (2015) Citoyens en danger contre riverains responsables. La mobilisation habitante engendrée par l'élaboration des PPRT. Les Cahiers de la sécurité industrielle, n5, Toulouse, Fondation pour une culture de sécurité industrielle.

MARTINAIS, Emmanuel, MOREL-JOURNAL, Christelle et DUCHÊNE, François (2006) La construction sociale du risque environnemental: un objet géographique? Dans Raymonde Séchet et François Duchêne (dir.) Penser et faire la géographie sociale. Contribution à une épistémologie de la géographie sociale. Rennes, Presses universitaires de Rennes, p. 173-186. 
MEAD, George Herbert (2006) L'esprit, le soi et la société. Suivi de trois essais. Paris, Presses universitaires de France.

PLATON, Jeannis Michail (2010) Descubra São Sebastião. São Paulo, Câmara Brasileira do Livro.

RAMIRES Jane Zilda dos Santos et RIBEIRO Wagner Costa (2011) Gestão dos riscos urbanos em São Paulo: as áreas contaminadas. Confins. Revue franco-brésilienne de géographie, vol. 13 [En ligne]. http://confins.revues. org/7323

SANTOS, Francine Modesto dos et MARANDOLA JUNIOR, Eduardo José (2012) Populações em situação de risco ambiental e vulnerabilidade do lugar em São Sebastião, Litoral de São Paulo. Desenvolvimento e Meio Ambiente, vol. 26, p. 103-125.

ZASK, Joëlle (2008) Questions environnementales et participation démocratique. Raison publique, $n^{\circ} 8$, avril, p. 43-55.

ZONABEND, Françoise (1993) Au pays de la peur déniée. Communications, vol. 57, n, p. 121-130. 